\title{
Macro-etching of Continuous Cast Steel
}

Jim Casey, Dofasco Inc. *

* Box 2460, Hamilton, Ontario, Canada, L8N 3J5

Investigations on service failures in line pipe steels have underscored the importance of control of macro-segregation during slab casting. In some investigations, crack initiation in line pipe service failures has been traced to martensitic or hard segregation bands outcropping on the pipe surface. The formation of a brittle martensitic band is due to a hardenability increase from Mn and carbon enrichment resulting from macro-segregation in the casting operation. Oxide and sulfide inclusions occur in the macro-segregated regions. Hydrogen diffusion to these inclusion sites in sourgas line pipe is known to cause catastrophic in-service failures. Segregation related problems are also known to affect the quality of the slab during the bending and unbending operation in the caster, resulting in problems with the seam welding of plates and hot rolled strip used to make pipes. Macrosegregation also affects subsequent field operations involving hot bending and field welding of pipes. Hence a method of preventing macro-segregation by fluid flow control in the caster, and having a method to monitor segregation have become an important quality assurance issue in the manufacture of line pipe and heavy plates. Since the base chemistry of the modern line pipe steel consists of ultralow sulfur $(<0.005 \mathrm{wt} \% \mathrm{~S})$ and low carbon $(<0.06 \mathrm{wt} \% \mathrm{C})$, the traditional Baumann sulfur printing method does not provide any useful information. Extensive research was carried out at Dofasco at the time of installation of their new caster to develop enhanced techniques for etching and documenting the macro-structure of low residual, as-cast slabs. Details of the techniques involving sample surface preparation, deep etching (using three different techniques) followed by ink highlighting will be outlined. The relative merits of documenting the resulting macrostructures using four different recording methods will be examined(i.e., taping, paper printing, Intaglio printing and digitized imaging).

The applications will be highlighted along with metallurgical case histories, to demonstrate the power of the techniques in problem solving. The first application is in characterization of macrosegregation and defects in as-cast slab hot-rolled and weld fabricated structures. The techniques were instrumental in establishing two underlying mechanisms, which initiated hook cracks in line pipe steel. The case histories will be presented. The second major application of the new techniques is in the identification of casting defects at the time of caster startup. Figure-1 shows a typical example of midway cracking, as well as centre-line segregation in a slab of low residual chemistry. Figure-2 shows a typical Intaglio print of a conventional HIC resistant line pipe slab showing equiaxed crystals nucleated by a falling shower of dendrites through the remelting phenomenon. Based on the detailed information derived from the macro-structure, the base chemistry, and the casting machine variables used to control the fluid flow, such as casting speed, superheat and soft reduction ratios, effective steps can be taken to eliminate macro-segregation in the slab. Figure -3 shows crystal structure details, along with the center line segregation present in a thin slab structure having a low residual chemistry. The paper will show that the techniques can be used for offline monitoring and control of macro-segregation in ultra-low residual chemistry steels.

With the emergence of thin strip casting, these techniques should prove valuable tools, in assisting metallurgists and production engineers to monitor and control the fluid flow and solidified structures encountered in the development of near net shape casting technology. 


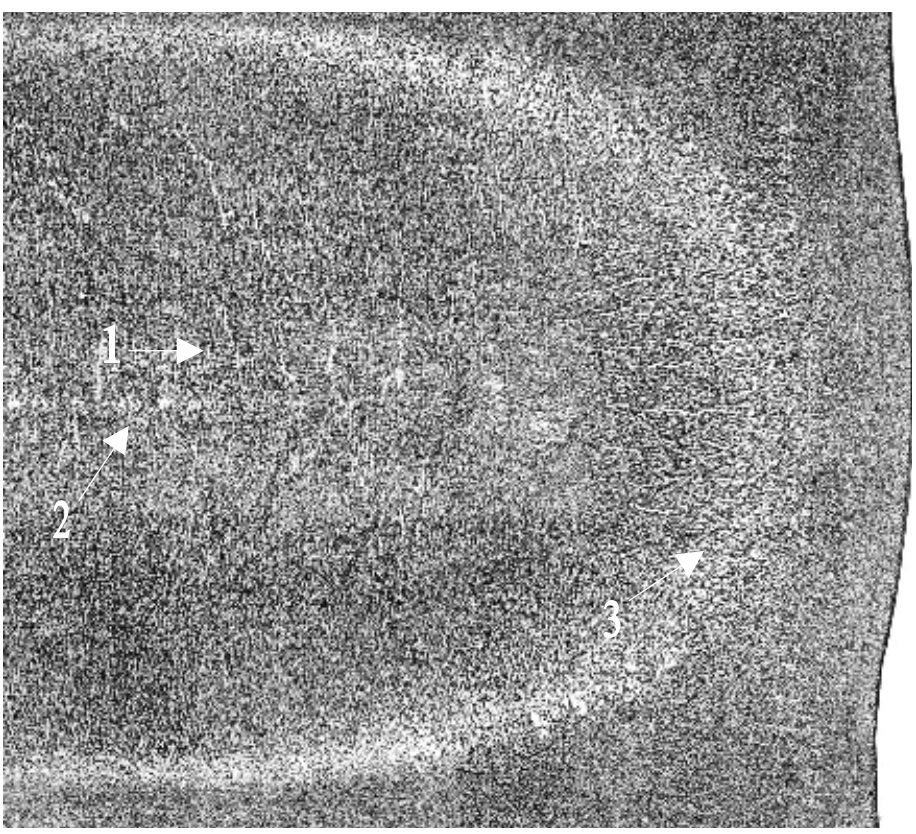

Figure 1: Relief Ink Print (X 0.35)

Continuous Cast Steel Slab, Transverse Section with moderately low residual chemistry*. Etched with hot HCL (50\% v/v at 90C) and printed using a

conventional relief ink printing method.

The structure reveals heavy mid way cracking (1), centerline (2) segregation and triple point cracks (3), not easily revealed by conventional sulphur prints.

* Analysis w\% --- C (0.30), Mn (0.53), Si (0.233), $\mathrm{S}$ (0.003), P (0.012), Al (0.058, Ca (0.029)

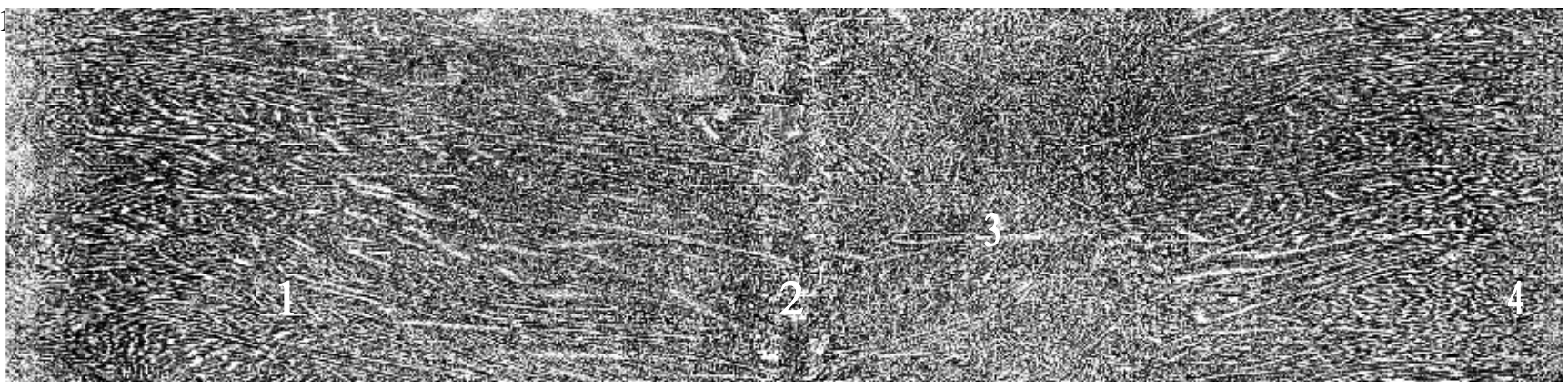

Figure 2: Intaglio Print (X 0.67)

Longitudinal section of a continuously cast, calcium treated, hydrogen induced cracking resistant line pipe steel, having a low residual chemistry. The steel is etched in two stages using Humfrey's Reagent. Neutral, 12\% Copper Ammonium Chloride, followed by subsequent immersion in acidified, (4\% v/v HCL), 12\% Copper Ammonium Chloride. Etching is performed at room temperature. The image is an Intaglio ink print, made using printing ink and a high pressure, mechanical printing press.

This "Copper" etch produces excellent resolution of the primary solidification structures on what is a very low residual chemistry steel *, revealing primary dendrites (1), centerline (2), equiaxed zone (3) and the chill zone (4). A second advantage of this technique is that the inclusions remain intact and are not etched out, as in hot acid etching, therefore allowing for subsequent probe analysis and chemical characterization when doing a forensic analysis on failed or rejected steel sections.

* Analysis w\% --- C (0.08), Mn (0.61), Si (0.214), S (0.0018), P (0.005), Al (0.075, Ca (0.0066)

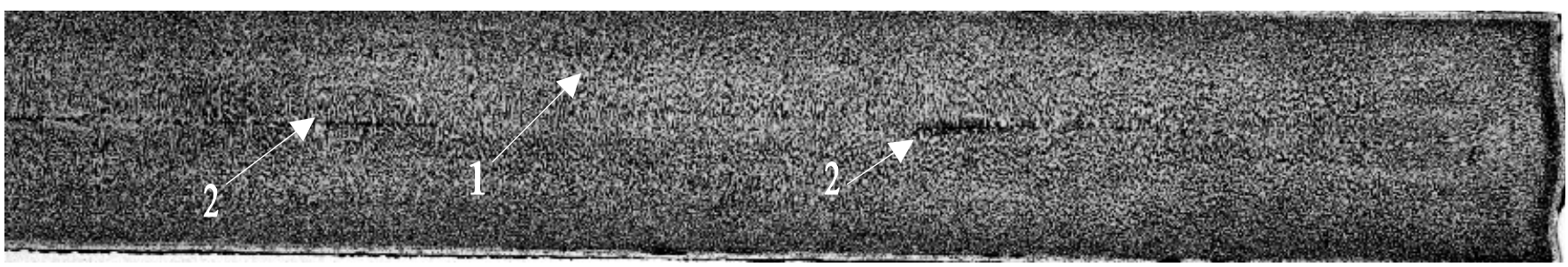

Figure 3: Intaglio Print (X 0.58)

Transverse section of a continuously cast thin slab with low residual chemistry *, etched using Humpfrey's Reagent. The fine primary dendrites (1) are easily revealed, as is solute segregation concentrated in patches along the centerline (2). Conventional etching in hot acid and the use of sulphur prints reveals nothing of the structure hidden within these thin as cast slabs.

* Analysis w\% --- C (0.059), Mn (0.30), Si (0.030), S (0.002), P (0.009). Al (0.035) 